\title{
Simultaneous Bilateral Femoral Neck and Proximal Humeral Fractures - Floating Members: A Case Report
}

\author{
Anderson Freitas ${ }^{1}$, Maxwell Oliveira ${ }^{1}$, Silvio L Macedo Neto ${ }^{1}$, Helio I \\ Costa ${ }^{1}$, Diogo R Souto', Laís Maria Pinto Almeida ${ }^{2}$, Fabrício Reichert \\ Barin $^{1 *}$ and Vincenzo Giordano ${ }^{3}$ \\ ${ }^{1}$ Instituto de Pesquisa e Ensino do Hospital Ortopédico e Medicina Especializada (IPE \\ - HOME), Brasília Distrito Federal, Brazil \\ ${ }^{2}$ Centro Universitário Tiradentes, Maceio/Alagoas, Brazil \\ ${ }^{3}$ Orthopedics and Traumatology Service Nova Monteiro - Miguel Couto Municipal \\ Hospital, Rio de Janeiro, Brazil \\ *Corresponding Author: Fabrício Reichert Barin, Instituto de Pesquisa e Ensino \\ do Hospital Ortopédico e Medicina Especializada (IPE - HOME), Brasília Distrito \\ Federal, Brazil.
}

\begin{abstract}
The first reports of simultaneous bilateral fractures of the femoral neck (BSFNF) and bilateral fractures and dislocations of the proximal humerus (BSPHFD), occurred in descriptions of complications in the treatment of psychiatric patients undergoing electroconvulsive therapy or by the use of drugs that induced seizures, since these caused strong muscle contractions, triggering, in some situations, simultaneous fractures.

These injuries are also seen in patients with osteometabolic diseases and in cases of accidents with electrocution. In the literature, few cases of BSFNF or BSPH were observed and their association was not found The authors will report the case of a 66-year-old man weighing 154 Pounds (70 kg) with four simultaneous injuries, BSFNF and BSFNF after an episode of seizure, describing what could be the first case of non-traumatic dissociation of all four members, so that we could call floating members.

The description of this case may guide future discussions about the need to perform diagnostic imaging of several joints in patients that are brought to the hospital after an episode of seizure, so that we can track more effectively the possibility of multiple fractures, as happened with the patient reported here.
\end{abstract}

Keywords: Humeral Fracture; Femoral Neck; Case Report; Bilateral

\section{Introduction}

The first reports of simultaneous bilateral fractures of the femoral neck (BSFNF) and simultaneous bilateral displacements of fracture of the humerus (BSPHFD), were described as resulting from the treatment of patients victims of epileptic seizures, however the simultaneous occurrence involving the four limbs, until the moment has no description [1].
These injuries are also seen in patients with osteometabolic diseases, such as renal osteodystrophy [2] and in cases of electrocution accidents. Cases of associated and concomitant fractures of femoral neck and proximal humerus are even more sparse. However, most part of the reports presents BSFNF or BSPHFD and the concomitance of these four injuries are even rarer [3]. Thus, the authors will report a case of four simultaneous fractures of both femoral necks and proximal humeri after a seizure episode, in the 
same patient, describing what could be the first case of non-traumatic dissociation of all limbs from the body, what we named as floating members.

\section{Case Presentation}

A 66-years old male patient was admitted in the Emergency Department brought by the EMS, with history of two consecutive episodes of seizures, one of them during the admission. After clinical stabilization, reported intense pain on both shoulder. As a non-trauma patient and having no other complains, shoulders radiographs were taken, revealing 4-part posterior fracture-dislocation of the right proximal humerus (Figure $1 \mathrm{~A}, \mathrm{~A}^{\prime}$ ) and posterior fracture-dislocation with a reverse Hill-Sachs lesion of the left shoulder (Figure 1B, B'). The patient was still under effects of anticonvulsant medication, poorly collaborative on the secondary evaluation after the imaging investigation. Family members were present and were asked about his previous medical conditions. They reported advanced Parkinson disease since 2009, with difficult control, using Prolopa BD 100/25mg, Prolopa HBS, Entarkin $200 \mathrm{mg} /$ day, Pramipexole $1 \mathrm{mg} /$ day and Rasagilin 1mg/day. Muscle spasms reduced overnight, there were no seizure episodes and the Neurology staff were against immediate surgical intervention. After fourteen days of intrahospital control of seisures, the patient was treated with simultaneous surgery of both shoulders (hemiarthroplasty of the right shoulder and McLaughlin procedure (lesser tuberosity transfer to the osseous depression and fixation with one $4 \mathrm{~mm}$ canulated screw) (Figure 2B).

It is important to mention that, even under supervision of physical therapists, nurses and medical doctors, in any moment the patient complained about pain in the lower limbs. Nevertheless, it was noted that the patient showed major impairment of body balance and disability to walk, initially atributed to Parkinson's disease. The patient himself and also the family members stated that, when the disease was uncontrolled, he was unable to walk. Although, the patient reported feeling comfortable when seated on the bed.

The patient returned on a routine appointment after 14 days, and still complaining inability to walk and pain on both hips. X-rays were taken, and revealed severe osteopenic bones bilateral femoral neck fracture (Garden 4 on both hips) (Figure 1C). The patient was readmitted immediately.
Clinical status got worse on the first day after admission, presenting dizzyness and dyspnea. The patient was underwent to a pulmonary angio-CT which showed pulmonary embolism (04/03/2017). Doppler scan of lower limbs revealed venous thromboembolism in the left tibialis vena of the left leg. After clinical stabilization, a vena cava filter was placed.

After meticulous clinical evaluation, the patient was eligible to definitive treatment. Simultaneous total hip replacement was proceeded under spinal anaesthesia and with vesical catheter, on lateral decubitus through a modified Hardinge approach (first the left hip). The implants used on the bilateral sides a uncemented hip arthroplasty (Figure 2A). After change of decubitus and new skin prep, the same approach was used on the right side. In each wound, a suction drain was left.

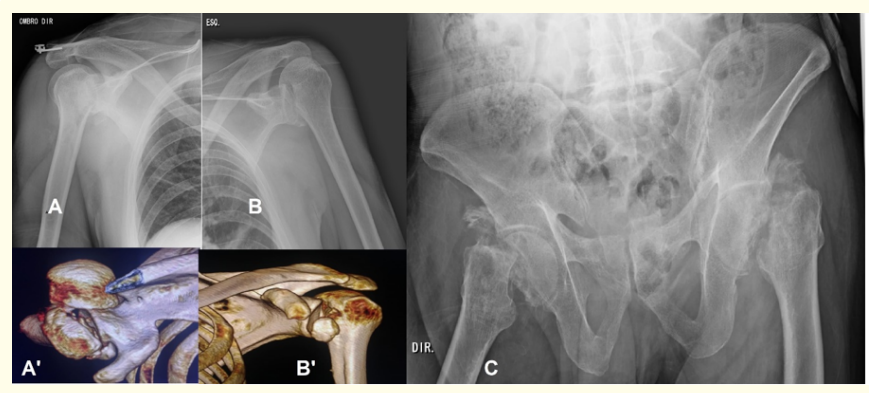

Figure 1: A: Radiographic image of the right shoulder with a four-part fracture. A': 3D tomographic image of the right shoulder with a four-part fracture. B: Radiographic image of the left shoulder with fracture and dislocation. B': 3D tomographic image of the left shoulder. C: panoramic radiograph of the pelvis showing fracture of both femoral necks.

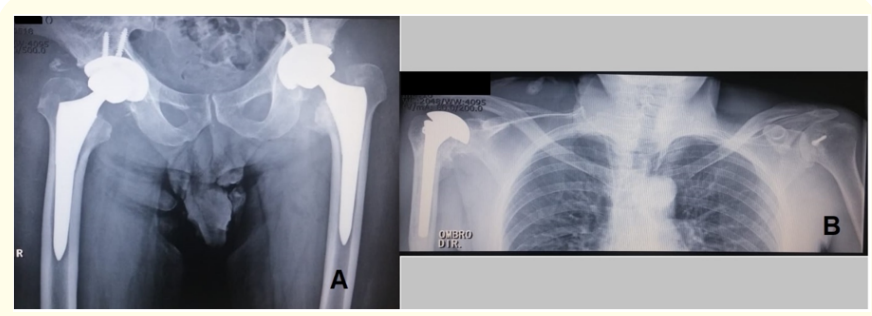

Figure 2: A: Panoramic radiograph of the pelvis in AP, showing bilateral uncemented arthroplasty of the hips. B: AP radiography of the shoulder girdle, showing partial arthroplasty of the right shoulder and a fixation screw for the fracture of the left shoulder. 
During the procedure, the patient received 3.5L of cristaloyds IV, with no need of blood transfusion. Immediate post operative care was taken at the ICU, with abduction device while at bed, mechanical prophylaxis for VTE with supervised physical therapy and intermittent pneumatic compression devices. Farmacological prophylaxis for VTE was made with Enoxaparin 40mg subcutaneously. After 24 hours, he was discharged from the ICU after removal of vesical catheter and the wound drains. At the ward, mechanical and farmacological measures were kept under supervision, and the patient was encouraged to walk using assistive ambulatory devices. Pain post operatively was mild, being handled with regular analgesic drugs.

Hospitaller discharge was granted after 5 post-op days, keeping VTE prophylaxis with Rivaroxaban $10 \mathrm{mg}$ /day during 30 days. On the 15th post-op day, the patient returned to follow-up; mobility and stability were successfuly tested. No readmissions or complications were observed during the first 36 months post-operative.

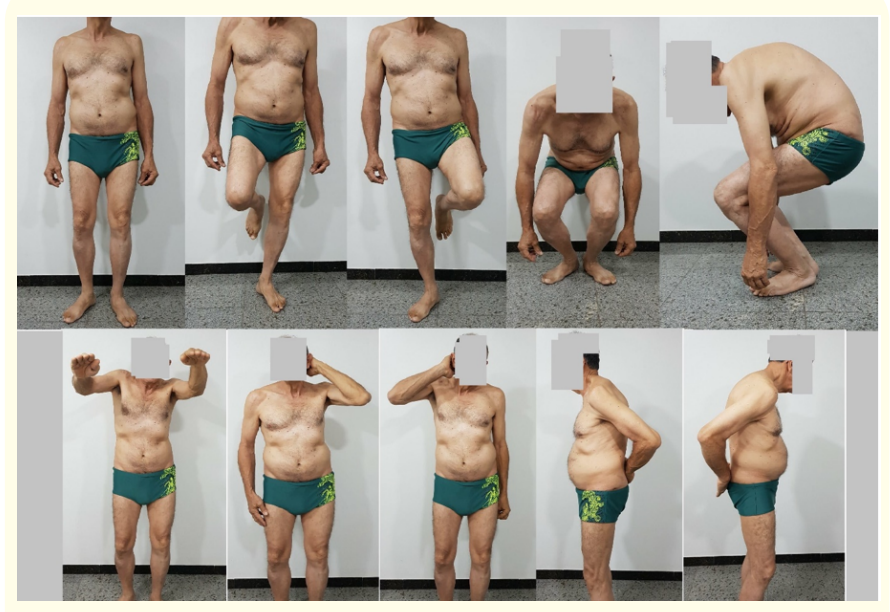

Figure 3: Upper: Photos of the functional assessment and degree of active mobility of the hips in orthostatism. Inferior:

Photos of the functional assessment and degree of active mobility of the shoulders.

\section{Discussion}

Technical advances in the medical field on the last century allowed a considerable raise in life expectancy, even in developing countries. Increase in the elderly people on the last decades led to a concomittant increase also in the hip fractures [4]. Femoral neck fracture is a common entity on the orthopaedic practice; however, bilateral femoral neck fractures are unsual events. Some reports indicate violent muscle contractions are the main cause for such injuries [1,5]. Bilateral simultaneous proximal humeral fractures are also uncommon events. The coexistence of bilateral proximal humeral fractures and bilateral femoral neck fractures makes this case an unique event.

Special attention must be taken to simultaneous BSFNF and BSPHFD, speccialy in pacientes victims of tonic-clonic seizures, due to the intimal relationship of this event. Diagnosis may be neglected because of irresponsive post epileptic status (dizzyness, mental confusion), multidisciplinary approach is essential in such cases, increasing the chances of successful evaluation.

\section{Conclusions}

Simultaneous BSFNF and BSPHFD are singular events. The paucitiy of cases in current medical literature makes difficult to stablish protocols for optimal medical care, forcing us to deal with such complex cases individually. The absence of guidelines demands thorough evaluation in order do reduce missing fractures, identify fractures with uncommon symptoms and conduct cases in fast and resolutive way, minimizing complications associated to a late diagnosis.

\section{Funding}

There is no funding to this study.

\section{Conflict of Interest Statement}

All authors declare that they have no conflict of interest.

All procedures followed were in accordance with the ethical standards of the responsible committee on human experimentation (institutional and national) and with the Helsinki Declaration of 1975, as revised in 2008. Informed consent was obtained from all patients for being included in the study. 


\section{Bibliography}

1. Powell HDW. "Simultaneous bilateral fractures of the neck of the femur". Journal of Bone and Joint Surgery [Br] 42B (1960): 236-252.

2. Zingraff J., et al. "Bilateral fracture of the femoral neck complicating uremic bone disease prior to chronic hemodialysis". Clinical Nephrology 2.2 (1974): 73-75.

3. Haronian E., et al. "Simultaneous bilateral femoral neck fracture and greater tuberosity shoulder fracture resulting from seizure". Orthopedics 25.7 (2002): 757-758.

4. Dedichen HH. "A Comparison of 1459 shock-treated and 969 nonshock-treated psychoses in Norwegian hospitals". Minutes Psychitrica et Neurologica, Supplementum Acta Psychiatrica Scandinavia (1946): 37.

5. Hendrik Jansen., et al. "Simultaneous posterior fracture dislocation of the shoulder following epileptic convulsion". Journal of Surgical Case Reports 2012.11 (2012).

\section{Assets from publication with us}

- Prompt Acknowledgement after receiving the article

- Thorough Double blinded peer review

- Rapid Publication

- Issue of Publication Certificate

- High visibility of your Published work

Website: https://www.actascientific.com/

Submit Article: https://www.actascientific.com/submission.php Email us: editor@actascientific.com

Contact us: +919182824667 